\title{
Detección de blancos moleculares en la ruta de señalización del fosfatidil-inositol en Leishmania spp. mediante herramientas bioinformáticas y de modelado matemático
}

\begin{abstract}
Valeria Velásquez, Rodrigo Ochoa, Carlos Muskus
Programa de Estudio y Control de Enfermedades Tropicales-PECET, Universidad de Antioquia, Medellín, Colombia

Introducción. La leishmaniasis es una enfermedad de gran impacto en la salud pública. La Organización Mundial de la Salud considera prioritaria la investigación orientada al desarrollo de medicamentos para su tratamiento. La exploración de la ruta del fosfatidil-inositol es interesante, ya que está implicada en la supervivencia del parásito mediante el control de la osmorregulación, el transporte a través de las membranas y la activación de diversos factores de transcripción.

Objetivo. Proponer blancos para el desarrollo de medicamentos contra la leishmaniasis mediante el análisis bioinformático y el modelado matemático de esta ruta.

Materiales y métodos. Se caracterizaron las proteínas pertenecientes a la ruta del fosfatidil-inositol en las bases de datos TriTrypDB y Pfam. Posteriormente, se hizo un análisis de similitud con las proteínas humanas mediante las herramientas InParanoid7 y OrthoMCL. Finalmente, se propuso un modelo booleano de la ruta, utilizando los programas PROMOT y CellNetAnalyzer.

Resultados. Se reconstruyó y se describió la ruta de señalización del fosfatidil-inositol en Leishmania spp. El análisis de similitud con proteínas humanas determinó la viabilidad de las proteínas pertenecientes a la ruta del fosfatidil-inositol como potenciales blancos moleculares. Los modelos matemáticos permitieron integrar los elementos de la ruta y predecir un efecto inhibidor. Se propusieron los siguientes blancos para el desarrollo de medicamentos: inositol-3-fosfato-5-fosfatasa, fosfatidilinositol-4-cinasa, fosfatidil-inositol-3,4,5-trisfosfato-3-fosfatasa, e inositol-polifosfato1P-fosfatasa.

Conclusiones. La ruta de señalización del fosfatidil-inositol aparece como una alternativa sólida desde el punto de vista del modelo cualitativo y a partir de las proteínas encontradas. Se identificaron posibles blancos de medicamentos contra la leishmaniasis. Posteriormente, se buscarán medicamentos contra las proteínas detectadas y se hará la validación experimental.
\end{abstract}

Palabras clave: leishmaniasis/terapia, fosfatidilinositoles, biología computacional, modelos matemáticos. doi: http://dx.doi.org/10.7705/biomedica.v35i2.2298

Detection of molecular targets on the phosphatidylinositol signaling pathway of Leishmania spp. through bioinformatics tools and mathematical modeling

Introduction: Leishmaniasis is a disease of high impact on public health. Research on drugs for its treatment is considered a priority by the World Health Organization. The phosphatidyl-inositol signaling pathway is interesting to explore because it is involved in the survival of the parasite, by controlling osmoregulation, transport through membranes, and activation of transcription factors.

Objective: To propose drug targets against the disease through bioinformatic analysis and mathematical modeling of this signaling pathway.

Materials and methods: The phosphatidyl-inositol pathway proteins were characterized through Pfam and TriTrypDB databases. Subsequently, a similarity analysis with human proteins was performed using the OrthoMCL and InParanoid7 tools. Finally, a boolean model of the pathway was proposed using PROMOT and CellNetAnalyzer softwares.

Results: The phosphatidyl-inositol signaling pathway in Leishmania spp. was reconstructed and described. The similarity analysis determined the feasibility of the phosphatidyl-inositol pathway proteins as molecular targets. Mathematical models allowed integrating the elements of the path and predicted an inhibitor effect. The following were proposed as drug targets: inositol-3-phosphate-5-phosphatase, phosphatidylinositol-4-kinase, phosphatidylinositol-3,4,5-trisphosphate 3-phosphatase and Inositol-1Ppolyphosphate phosphatase.

\section{Contribución de los autores:}

Valeria Velásquez: análisis bioinformático y construcción del modelo matemático

Rodrigo Ochoa y Carlos Muskus: asesoría y revisión del manuscrito final 
Conclusion: The phosphatidyl-inositol signaling pathway is robust from the point of view of the qualitative model and the proteins found. Thus, potential drug targets against leishmaniasis were identified. Subsequently we will seek to detect drugs against this set of proteins and validate them experimentally.

Key words: leishmaniasis/therapy, phosphatidylinositols, computational biology, mathematical models. doi: http://dx.doi.org/10.7705/biomedica.v35i2.2298

La leishmaniasis es una enfermedad cuyo impacto en la salud pública ha sido subestimado por la carencia de políticas de control y prevención en los países en los que se presenta, lo cual se ve reflejado en la indiferencia farmacéutica. La población en riesgo de contraer leishmaniasis se ha estimado en 350 millones de individuos en 98 países. En los últimos diez años, las regiones endémicas se han extendido, con un fuerte incremento en el número de casos registrados de la enfermedad (1). La Organización Mundial de la Salud (OMS), en colaboración con diferentes organizaciones no gubernamentales (ONG), ha declarado como prioridad la investigación y el desarrollo de nuevos tratamientos anti-Leishmania que sean accesibles, seguros, eficientes, de corta duración, fáciles de administrar y a un costo razonable, con el fin de mejorar la calidad de vida de los pacientes que padecen esta enfermedad.

Una de las estrategias desarrolladas para la búsqueda de nuevos medicamentos consiste en el modelado o reconstrucción de vías metabólicas importantes que faciliten análisis bioquímicos detallados en el organismo de interés, permitiendo así la identificación de posibles blancos moleculares específicos para estos medicamentos (2-4). Los resultados obtenidos en los últimos años en la exploración de la vía del metabolismo del fosfatidil-inositol la postulan como una alternativa en el desarrollo de medicamentos para diversas enfermedades, incluida la leishmaniasis (5-9). Esta vía resulta interesante debido a la gran cantidad de procesos intracelulares que controla, los cuales van desde la endocitosis y la remodelación del citoesqueleto, hasta la activación de factores de transcripción, proliferación, diferenciación y crecimiento celular. En diversos estudios de sobreexpresión o inhibición de algunas proteínas

\footnotetext{
Correspondencia:

Carlos Muskus, Sede de Investigación Universitaria, Universidad de Antioquia, Calle $62 \mathrm{~N}^{\circ}$ 52-59, torre 2, laboratorio 632, Medellín, Colombia Teléfono: (574) 219 6507; fax: (574) 2196511

carmusk@yahoo.com
}

Recibido: 18/02/14; aceptado: 12/02/15 pertenecientes a esta ruta de señalización en Trypanosoma spp. (un género de organismos filogenéticamente muy relacionados con Leishmania spp.), se han encontrado serias alteraciones en la morfología y fisiología de este parásito, entre las que se encuentran cambios en el citostoma y el bolsillo flagelar, en el trasporte a través de la membrana, en el crecimiento celular y en el remodelado del citoesqueleto, así como una disminución en la proliferación y daños celulares en la citocinesis y la endocitosis (5,10-13).

Además, esta ruta se ha descrito casi totalmente en todos los grandes grupos eucariotas, según se constata en la base de datos KEGG (Kyoto Encyclopedia of Genes and Genomes) (14), lo que constituye una ventaja, ya que son muy pocos los datos sobre otras rutas promisorias. Con base en esta información, se hizo un análisis bioinformático de las proteínas pertenecientes a la ruta de señalización y se planteó un modelo matemático que describe las interacciones de los elementos pertenecientes a la vía y predice los posibles efectos en sus alteraciones, especialmente el efecto inhibidor, el cual constituye la vía de acción de la mayoría de los medicamentos en múltiples enfermedades. Con ello, se pudieron identificar potenciales blancos moleculares para el desarrollo de medicamentos para la leishmaniasis, que pueden evaluarse en modelos in vitro e in vivo.

\section{Materiales y métodos \\ Obtención de secuencias}

Primero se buscaron todas las proteínas relacionadas con la ruta de señalización del fosfatidilinositol en Leishmania spp., a partir de los datos reportados en la KEGG (14). Posteriormente, se buscaron estas proteínas en la base de datos TriTrypDB (versión 4.0) (15), empleando el genoma de Leishmania major. Los ortólogos de todos los genes presentes en la KEGG e identificados inicialmente en L. major, se buscaron en otras especies del género Leishmania cuyo genoma estuviera secuenciado empleando el programa Protein Basic Local Alignment Search Tool (BLASTP) (16). Por último, en cada una de las especies de Leishmania se comprobaron las proteínas relacionadas con secuencias disponibles 
que, hasta el momento, son las de L. major, $L$. donovani, L. infantum, L. braziliensis, L. mexicana y L. tarentolae.

\section{Búsqueda de dominios}

En todas las proteínas de la ruta del fosfatidilinositol y en las proteínas relacionadas, se buscaron los dominios presentes en cada secuencia, utilizando la base de datos Pfam (17), la cual hace búsquedas de patrones específicos en secuencias de aminoácidos mediante la aplicación de las cadenas de Markov. Con la información obtenida, se depuraron las secuencias y se incluyeron solo las proteínas funcionales desde el punto de vista de la arquitectura de dominios, descartando las proteínas no funcionales o seudogenes. Posteriormente, se construyó un modelo cualitativo de la ruta del fosfatidil-inositol a partir de las proteínas encontradas en cada especie analizada de Leishmania.

\section{Análisis de similitud con las proteínas de la vía del fosfatidil-inositol en humanos}

Se calculó la similitud y se constató la presencia o ausencia de ortología de la secuencia de los genes de la ruta en Leishmania spp., y los resultados se contrastaron con las respectivas proteínas del humano. Para ello, se hizo una búsqueda de proteínas ortólogas con la herramienta OrthoMCL (18) de la base de datos TriTrypDB (versión 4.2) (19) y la base de datos InParanoid 7 (20). Posteriormente, se hizo una DELTA-BLAST (Domain Enhanced Lookup Time Accelerated BLAST) (21) de las secuencias representantes de cada gen y, en la base de datos de proteínas humanas, se compararon los parámetros y se identificaron las proteínas con menor similitud y nivel de identidad.

\section{Construcción del modelo matemático booleano de la ruta}

En esta fase se construyó un modelo booleano de la ruta de señalización del fosfatidil-inositol, a partir de las interacciones reportadas en la literatura científica (5,22-29), así como la información de las proteínas encontradas para Leishmania spp. Los datos recolectados permitieron inferir la activación de diversos factores de transcripción, y de procesos de proliferación, crecimiento y supervivencia. Este procedimiento se hizo con el programa ProMoT, versión 8.5 (30). El resultado, es decir, la descripción gráfica y matemática del modelo, se exportó al paquete de análisis de redes biológicas CellNetAnalyzer (CNA) (22), con el que se hicieron todas las simulaciones del modelo booleano.
Desde el punto de vista matemático, cada interacción se expresa con un lenguaje que representa y formaliza la red de una manera adecuada, mediante el uso de los operadores booleanos "Y" (?), O (+) y NO (!), que son suficientes para representar cualquier relación lógica, convirtiéndose en las reglas generales que regirán el funcionamiento del modelo. Así, el algoritmo toma cada nodo desde los estímulos externos del modelo y calcula el estado de activación o desactivación de cada uno, con base en las relaciones lógicas que los definen. Esas relaciones lógicas involucran varios nodos y muestran cómo se produce su activación o desactivación: en el caso más simple, se encuentra una interacción de activación uno a uno, es decir, una molécula activa a otra, por ejemplo, la activación por fosforilación de una cinasa. Existen otros casos en los que las relaciones son más complejas, como el de un nodo que puede ser activado por más de una molécula, o que es activado por una y desactivado por otra; esos eventos de señalización se representan mediante un conector de tipo "O" y se distinguen según su efecto de inhibición o activación.

\section{Simulaciones de la red booleana}

Para la simulación, los estímulos externos que activan algunas cinasas y fosfatasas, las cuales representan la entrada de la red configurada (en estado activo o inactivo en cada uno de estos nodos), se muestran en todos los casos analizados (figuras 1 y 2). Sin embargo, su elección se establece de forma precisa, debido a que los mecanismos exactos que desencadenan estas cascadas en Leishmania spp. aún no son claros. La respuesta de la red se midió con respecto a la activación de las respuestas mediadas por los segundos mensajeros: el fosfatidil-inositol 3 fosfato (PI3P), que controla el transporte a través de membranas; el fosfatidil-inositol $3,4,5$ trifosfato $(\mathrm{PI}, 4,5 \mathrm{P} 3)$ y el fosfatidil-inositol 4,5 difosfato (PI4,5P2), los cuales, según se ha demostrado, controlan la activación de factores de transcripción, y el diacilglicerol, el cual controla la activación de la ruta del calcio hasta la proteína cinasa $\mathrm{C}$.

Posteriormente, se hicieron variaciones en la señal de entrada de la red para observar los efectos en la respuesta con el modelo asincrónico, separando las fases temporales.

\section{Evaluación del efecto de un inhibidor sobre la ruta}

Después de la evaluación exploratoria de la regulación de la ruta modelada en el enfoque booleano, 


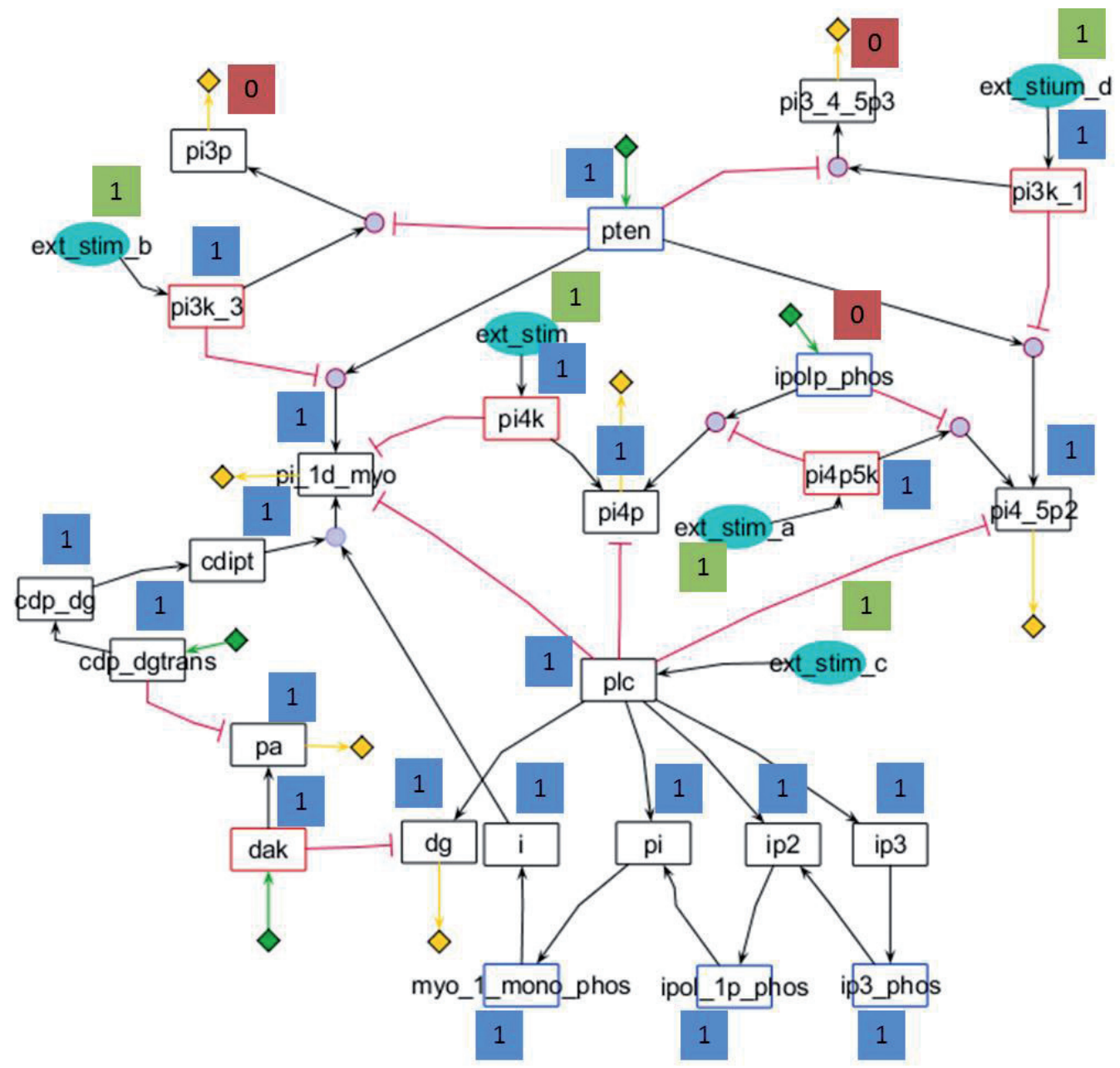

Figura 1. Simulación de los eventos tardíos de la ruta del fosfatidil-inositol. Se muestra la activación de los nodos en azul (1) y la desactivación en rojo (0). Los estímulos de entrada se muestran en verde (1). Además de las proteínas ya presentadas en el cuadro 1, los metabolitos y estímulos presentes son: PI3_4_5P3 (fosfatidil-inositol 3,4,5 trifosfato), PI3_4P2 (fosfatidil-inositol 3,4 difosfato), PI4P (fosfatidil-inositol 4 fosfato), PI_1D_myo (fosfatidil-inositol 1D mioinositol), IP3 (inositol 3 fosfato), IP2 (inositol 2 fosfato), PI (inositol fosfato), I (inositol), DG (diacilglirerol), PA (ácido fosfatídico), y ext_stim (estímulos externos). Debido a restricciones del programa utilizado, fue necesario nombrar los nodos con guiones bajos (_) en vez de comas (,).

se simularon efectos inhibitorios sobre algunas proteínas de la ruta. Para ello, se calculó la matriz de dependencias en el programa CellNetAnalyzer, la cual relaciona cada especie del modelo con sus interacciones de activación, de desactivación, o con ambas. Después, se postularon las proteínas a las que se les aplicó un estado de apagado o desactivación en el modelo y se simuló dicho efecto sobre la red.

\section{Construcción del modelo lógico dinámico}

A partir del modelo booleano construido en CellNetAnalyzer, se utilizó el programa Odefy (31) para transformar el modelo en un sistema dinámico y, de esta manera, obtener información sobre los niveles de acumulación de los metabolitos en la ruta. Para ello, se utilizó la aproximación Hillcube, dejando los parámetros $\mathrm{K}=0,5$ y $\mathrm{t}=1$ constantes (constante de afinidad y tiempo de vida de cada especie, respectivamente) para todos los nodos con sus valores por defecto, que es lo recomendado cuando no se tiene información adicional. Este método utiliza la ecuación general de la red booleana determinada por la siguiente función: $x_{i}(t+1)=B_{i}\left(x_{i 1}(t), \ldots, x_{i N i}(t)\right) \varepsilon\{0,1\}, i=1,2, \ldots, N$, donde $\mathrm{N}$ es el número de especies $X_{i}$ que toman un valor xi $[0,1]$, y la transforma en un sistema de ecuaciones diferenciales, así: $\overline{\mathrm{X}}_{i}=\frac{1}{\tau}\left(\overline{\mathrm{B}}_{i}\left(\overline{\mathrm{x}}_{\mathrm{i1}}, \overline{\mathrm{X}}_{\mathrm{i12}}, \ldots, \overline{\mathrm{X}}_{i N i}\right)-\overline{\mathrm{X}}_{\mathrm{i}}\right)$, donde se muestra la tasa de producción de la especie $X_{i}$ en un tiempo $t$, mediante una ecuación de decaimiento de primer orden, determinada por el tiempo de vida de 


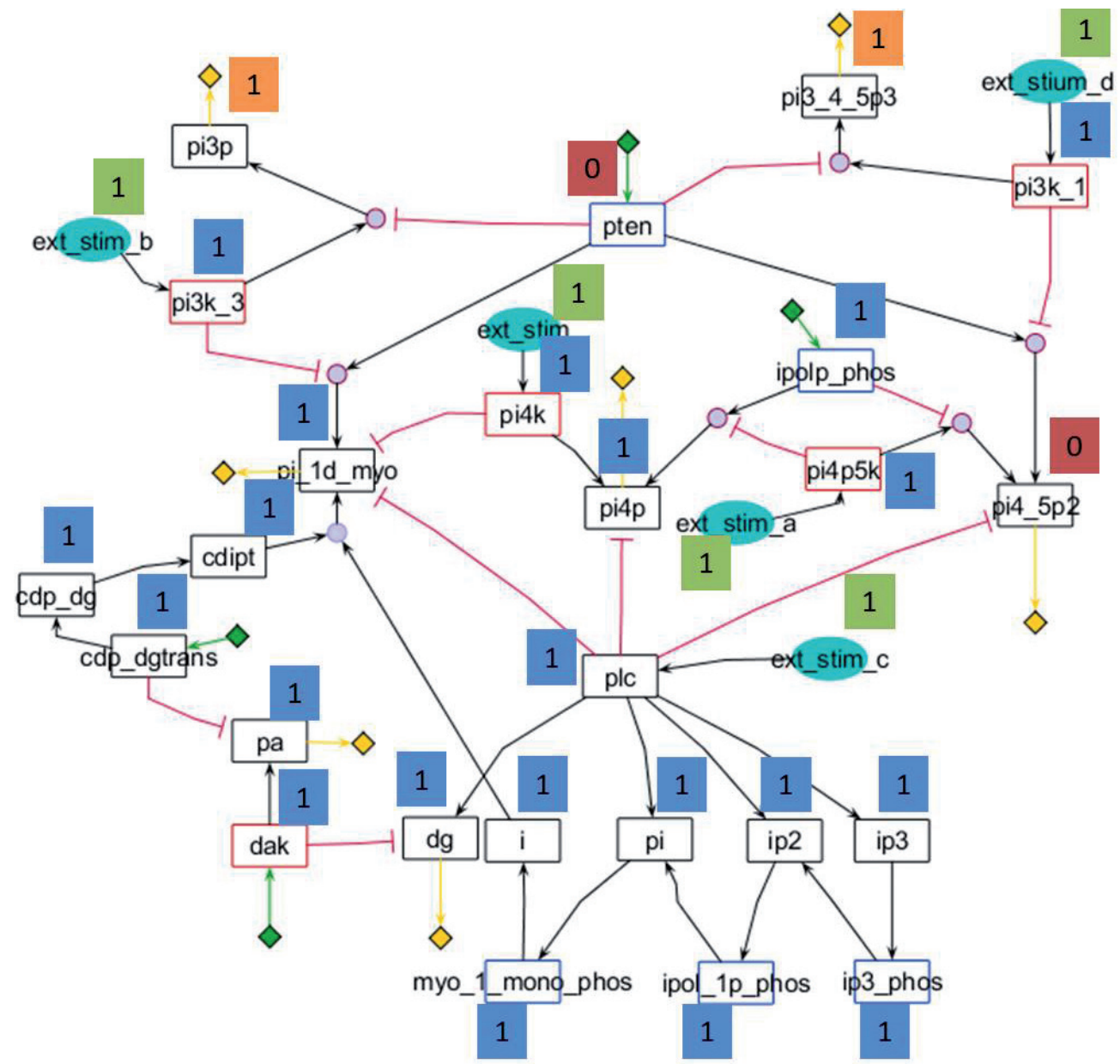

Figura 2. Efecto de la inhibición de la proteína PTEN sobre la ruta del fosfatidil-inositol. La configuración de la ruta se mantuvo en estado tardío (tal y como se muestra en la figura 1). Al desactivar la proteína PTEN (indicada con un recuadro en rojo), se produce la desactivación del metabolito PI4_5P2 (también en rojo) y una desregulación de PI3P y PI3_4_5P3 (marcados con recuadros naranja). Los demás nodos de la red permanecieron sin alteraciones.

la especie i (Ti), y con la función continua $B$ dada por $\overline{\mathrm{B}}_{\mathrm{H}}\left(\overline{\mathrm{x}}_{1}, \overline{\mathrm{x}}_{2}, \ldots, \overline{\mathrm{x}}_{N}\right)=\overline{\mathrm{B}}_{1}\left(f_{1}\left(\overline{\mathrm{x}}_{1}\right), f_{2} \overline{\mathrm{x}}_{2}, \ldots, f_{N} \mathrm{x}_{N}\right)(31)$.

Para la simulación, se designaron diez unidades temporales, con base en las cuales se obtuvo la gráfica representativa de la dinámica de la red. Los resultados se compararon con los datos previamente obtenidos en la red booleana.

\section{Resultados}

Proteínas de Leishmania spp. para la ruta del fosfatidil-inositol

A partir de los análisis bioinformáticos iniciales, se describieron las proteínas cinasas y fosfatasas pertenecientes a la ruta de señalización del fosfatidil-inositol en Leishmania spp., con su nombre completo, su nombre abreviado y su clasificación según la Enzyme Commission (EC) (cuadro 1). Algunas de las proteínas encontradas no estaban en la clasificación de la EC y, además, no se encontraban en la ruta reportada en la base de datos KEGG. Pese a que la búsqueda inicial de dichas proteínas se hizo en esta base de datos, pesquisas más minuciosas en otras bases de datos llevaron a la identificación de otras. Sin embargo, las que se encontraron en la base de datos TriTrypDB, se mantuvieron para los análisis posteriores.

Con toda la información se construyó un modelo cualitativo inicial de las proteínas de la ruta en estas especies. Al comparar este modelo con la ruta general, se detectaron partes que no se encuentran activas en Leishmania spp., como la producción de fosfatidil-inositol-5-fosfato, o de fosfatidil-inositol-3,4-di-fosfato (PI3,4P2), por lo que se vería afectada la activación de procesos celulares asociados tradicionalmente a esta parte 
Cuadro 1. Enzimas encontradas para la ruta del fosfatidil-inositol en Leishmania spp. (se da el nombre abreviado, el nombre completo y la clasificación EC de aquellas que la tienen).

\begin{tabular}{|c|c|c|}
\hline Proteína & Nombre completo & EC \\
\hline $\mathrm{Pl} 4 \mathrm{~K}$ & Fosfatidil-inositol 4 cinasa & [EC:2.7.1.67] \\
\hline $\mathrm{PI}(4) \mathrm{P} 5 \mathrm{~K}$ & 1-Fosfatidil-inositol 4 fosfato 5 cinasa & [EC:2.7.1.68] \\
\hline PI3K Tipo $(1 \mathrm{~A}, 1 \mathrm{~B}, 2,3)$ & Fosfatidil-inositol 3 cinasa & [EC:2.7.1.137 \\
\hline PTEN (IP3 fos) & Fosfatidil-inositol $3,4,5$ trifosfato 3 fosfatasa & [EC: 3.1 .3 .48$]$ \\
\hline Myo- I- mono fos & Mio-inositol-1(o 4)-monofosfatasa & [EC:3.1.3.25] \\
\hline DAK & Diacil-glicerol cinasa & {$[E C: 2.7 .1 .107$} \\
\hline IP3 5 fos $\left(1 Y_{2}\right)$ & Inositol $1,4,5$ trifosfato 5 fosfatasa & {$[E C: 3.1 .3 .56]$} \\
\hline $\mathrm{PI} 3 \mathrm{~K} 2$ & Fosfatidil-inositol 4,5 bifosfato 3 cinasa & {$[E C: 2.7 .1 .153$} \\
\hline INNP (Ipol 1P fos) & Inositol-polifosfato 1 fosfatasa & [EC:3.1.3.57] \\
\hline $\mathrm{PI}(3,5) \mathrm{K}$ & Fosfatidil-inositol 3,5 cinasa & {$[E C: 2.7 .1 .150$} \\
\hline IpolP K/IP3 3K & Inositol-polifosfato cinasa & \\
\hline PIK & Fosfatidil-inositol cinasa & \\
\hline Ipol P fos & Inositol-polifosfato 5 fosfatasa & {$[E C: 3.1 .3 .36]$} \\
\hline PLC & Fosfatidil-inositol-fosfolipasa C & {$[E C: 3.1 .4 .11]$} \\
\hline CDIPT (CDP-DGI) & CDP-diacilglicerol-3 inositol-fosfatidil-transferasa & [EC:2.7.8.11] \\
\hline CDP-DG & Fosfatidato-citidil-transferasa & [EC:2.7.7.41] \\
\hline CALMODULINA & Calmodulina & \\
\hline PKC & Proteína cinasa $\mathrm{C}$ & {$[E C: 2.7 .11 .13]$} \\
\hline IP3R & Receptor de inositol 1,4,5-trifosfato & \\
\hline
\end{tabular}

de la ruta de señalización, los cuales aún no se han determinado claramente en los modelos en los que la ruta ha sido estudiada.

Después, fue posible identificar las copias de cada uno de los genes que codifican para las proteínas de la ruta del fosfatidil-inositol en Leishmania spp. Algunas proteínas resultaron codificadas por una serie de genes parálogos dentro de una misma especie, y conservaron su sintenia (fosfatidil-inositol 4 cinasa -PI4K-; fosfatidil-inositol 4 fosfato 5 cinasa -PI(4)P5K-; PI3K de tipo 1A, 1B, 2, 3; mio-inositol 1 monofosfatasa -mio-I-mono fos-; diacil-glicerolcinasa -DAK-; inositol 1,4,5 trifosfato 5 fosfatasa -IP3 5 fos-; fosfatidil-inositol-fosfolipasa C -PLC-, y fosfatidato-citidil-transferasa-CDP-DG-).

Al buscar los dominios, se destacaron la familia de proteínas fosfatidil-inositol cinasas (PIK) que contienen el dominio PIK (dominio catalítico), y una serie de variaciones de dominios que dan pistas sobre sus funciones celulares de manera más detallada. Entre el grupo de las fosfatasas, se destacó la proteína fosfatidil-inositol -3,4,5trifosfato 3-fosfatasa (PTEN), que actúa como fosfatasa en pasos diferentes de la ruta y a la cual se le encontraron dos dominios en la base de datos Pfam: DSPc y PTEN-C2.

\section{Análisis de similitud con las proteínas de la ruta del fosfatidil-inositol en humanos}

En la búsqueda se encontró un ortólogo en humanos para al menos un representante de las secuencias de la ruta, excepto para los genes INNP y $P I(3)$ $P 5 K$, lo que no significa que dichas proteínas no existan en el humano, pues estas búsquedas implican resultados por homología con base en un perfil filogenético, por lo que no debe descartarse una homología funcional, como se comprobó al examinar el mapa de la ruta en la base de datos KEGG y al utilizar la DELTA-BLAST.

A partir de los resultados de la DELTA-BLAST (cuadro 2), la cual se ejecutó para un representante de cada grupo de secuencias de las proteínas encontradas en la ruta de señalización, se encontraron proteínas con altos porcentajes de identidad y un bajo valor $\mathrm{E}$ de corte (menor de $1 \times 10^{-3}$ ), es decir, alineamientos con gran similitud y con pocas probabilidades de darse por el azar; otros alineamientos presentaron menores porcentajes de identidad y bajos valores $E$, con variaciones que permitieron proponer como posibles blancos de medicamentos a la proteína INNP (la cual ya había sido identificada en el análisis anterior) y a la fosfatasa IP35 fos, con el fin de evitar efectos secundarios graves en el humano. Esta fosfatasa cumple la función de desfosforilación de los mioinositoles trifosfato, durante su proceso de reciclaje para la formación de nuevos fosfatidil-inositoles.

\section{Análisis del modelo booleano}

El modelo booleano permitió describir lógicamente diferentes situaciones de la red y, por ende, predecir su respuesta en términos de activación o desactivación de los nodos correspondientes. 
Cuadro 2. Resultado de la DELTA-BLAST para las principales proteínas cinasas y fosfatasas de la ruta del fosfatidil-inositol en Leishmania spp. relacionadas con el porcentaje de identidad y el valor $\mathrm{E}$ (se muestran las tres primeras coincidencias de cada análisis).

\begin{tabular}{|c|c|c|c|}
\hline $\begin{array}{l}\text { Nombre del gen y secuencia } \\
\text { usada en el análisis }\end{array}$ & Secuencias encontradas (ID) & $\begin{array}{l}\text { Porcentaje de } \\
\text { identidad }\end{array}$ & Valor E \\
\hline$I P(3) 5$ phos & gi|301598678|pdb|3N9V|A & 15,92 & $3,00 \mathrm{E}-39$ \\
\hline \multirow[t]{2}{*}{ LbrM.11.0810 } & gi|299856846|pdb|3MTC|A & 15,92 & $3,00 E-39$ \\
\hline & gi|194384652|dbj|BAG59486.1| & 18,63 & $4,00 \mathrm{E}-39$ \\
\hline INNP & gi|119613713|gb|EAW93307.1| & 19,94 & $9,00 \mathrm{E}-51$ \\
\hline \multirow[t]{2}{*}{ LbrM.31.3290 } & gi|17389533|gb|AAH17801.1| & 19,35 & $1,00 \mathrm{E}-50$ \\
\hline & gi|116812595|ref|NP_006076.4| & 19,94 & $5,00 \mathrm{E}-50$ \\
\hline PI(3)P5K & gi|4589606|dbj|BAA76825.1| & 25,94 & $3,00 \mathrm{E}-87$ \\
\hline \multirow[t]{2}{*}{ LmjF.27.0890 } & gi|119582870|gb|EAW62466.1| & 18,16 & $6,00 \mathrm{E}-82$ \\
\hline & gi|21749863|dbj|BAC03674.1| & 25,62 & $7,00 \mathrm{E}-82$ \\
\hline Pl(4)P5K & gi|208431776|ref|NP_001129109.1| & 27,87 & $3,00 \mathrm{E}-133$ \\
\hline \multirow{2}{*}{ LmxM.33.3090 } & gi|4505815|ref|NP_003548.1| & 27,87 & $2,00 \mathrm{E}-132$ \\
\hline & gi|208431778|ref|NP_001129110.1| & 27,87 & $3,00 \mathrm{E}-132$ \\
\hline PI3K & gi|194391206|dbj|BAG60721.1| & 42,64 & $7,00 \mathrm{E}-106$ \\
\hline \multirow{2}{*}{ LbrM.24.2090 } & gi|194391206|dbj|BAG60721.1| & 22,34 & $2,00 \mathrm{E}-58$ \\
\hline & gi|194391206|dbj|BAG60721.1| & 21,55 & $1,00 \mathrm{E}-21$ \\
\hline PI4K & gi|32425423|gb|AAH18120.2| & 25,77 & 0.0 \\
\hline \multirow[t]{2}{*}{ LmjF.29.1450 } & gi|32197216|gb|AAH53654.1| & 25,67 & 0.0 \\
\hline & gi|4505807|ref|NP_002641.1| & 30,41 & 0.0 \\
\hline PLC & gi|14249340|ref|NP_116115.1| & 31,00 & 0.0 \\
\hline \multirow[t]{2}{*}{ LbrM.22.1590 } & gi|62897967|dbj|BAD96923.1| & 31,00 & 0.0 \\
\hline & gi|119591033|gb|EAW70627.1| & 31,30 & 0.0 \\
\hline PTEN & gi|118764003|gb|AAI28147.1| & 28,09 & $5,00 \mathrm{E}-49$ \\
\hline \multirow[t]{2}{*}{ LbrM.14.1280 } & gi|213972593|ref|NP_001135440.1| & 28,09 & $7,00 \mathrm{E}-49$ \\
\hline & gi|213972589|ref|NP_570141.3| & 28,09 & $1,00 \mathrm{E}-48$ \\
\hline
\end{tabular}

Esta simulación se dividió en dos momentos: una activación temprana de las cinasas y una activación tardía de las fosfatasas (figura 1). Después de la simulación, se encontró que el evento temprano permitió la activación de los segundos mensajeros PI3P y PI3,4,5P3, pero no de PI4,5P2, ya que este es consumido como sustrato por la enzima PI3K, y por lo tanto, es necesario conocer con mayor detalle la cinética de estas proteínas para determinar cuál es la relación entre la activación de PI3K y la producción de PI4,5P2. Una situación similar se presentó con la activación de PI(4)P5K, la cual se traduce en el consumo de fosfatidilinositol 4 fosfato (PI4P).

Al analizar el modelo con los eventos tardíos (segundo caso), se observó que la activación de las fosfatasas dirigió la producción diferencial de segundos mensajeros; por ejemplo, al activarse la fosfatidil-inositol 3,4,5 trifosfato 3 fosfatasa (PTEN), se redujo la producción de PI3P y $\mathrm{PI}, 4,5 \mathrm{P} 3$, potenciando la producción de PI4,5P2 y de PI4P indirectamente. Esta simulación tardía de la ruta se muestra en la figura 3 , en la cual se ve cómo la ruta recuperó el estado basal de activación después del estímulo, lo cual es muy importante en su regulación. De esta manera, fue posible constatar la importancia de la proteína PTEN como centro de la regulación de los fosfatidil-inositoles ya mencionados, y, por consiguiente, su importancia en la ruta de señalización, lo cual, sumado al conocimiento de su rol como gen supresor de tumores en otros modelos en mamíferos, la perfilan como un buen blanco para el desarrollo de medicamentos contra la leishmaniasis.

\section{Efecto de un modulador}

Con el fin de proponer posibles blancos de medicamentos a partir de la maximización del efecto de una molécula externa sobre la ruta de señalización, se construyó una matriz de dependencia entre las especies (figura 2). Los resultados indicaron que las especies que tienen un mayor número de interacciones con otros elementos de la ruta, son la hidrolasa PLC, y las fosfatasas IP35fos y PTEN, las cuales juegan un papel crucial en la regulación de la ruta. Al analizar en detalle cada caso y contrastarlo con los análisis de homología y similitud de las proteínas de la ruta de señalización en humanos, se encontró que la IP3 5 fos tenía el menor porcentaje de identidad con 


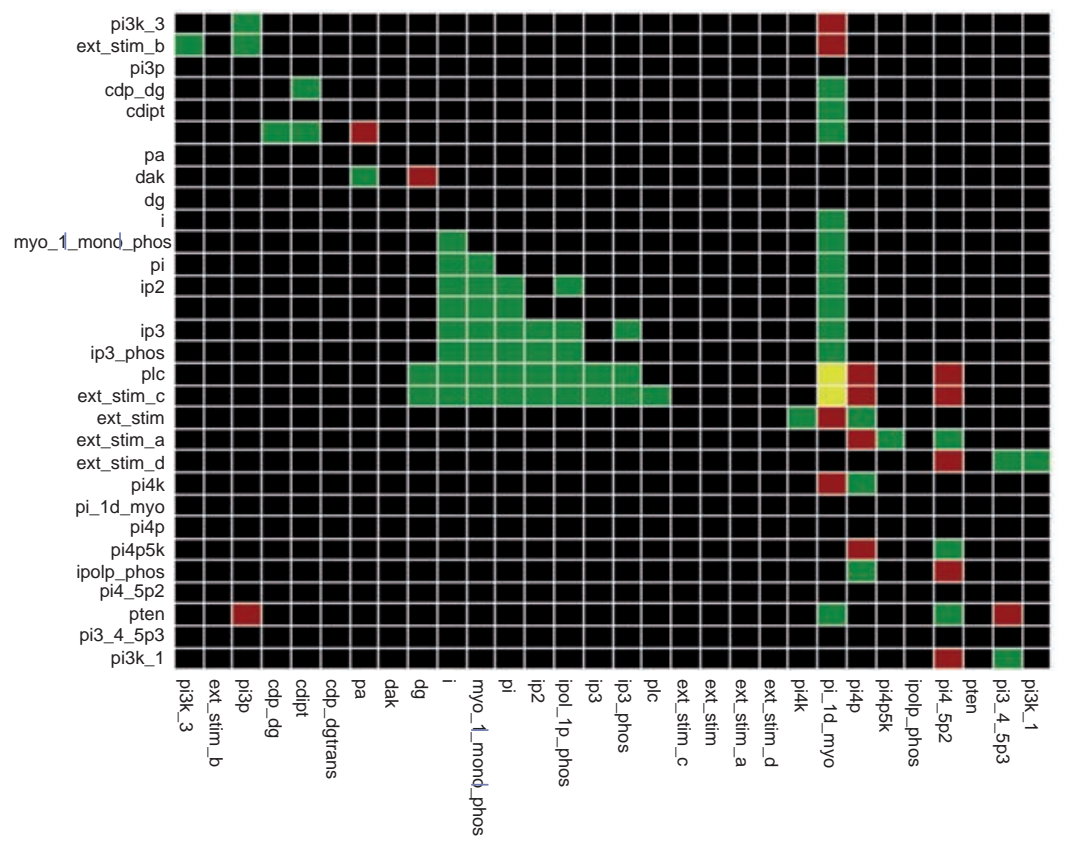

Figura 3. Matriz de la dependencia entre las especies relacionadas en el modelo cualitativo. En verde se muestra la activación de un nodo de la ruta (que puede ser una proteína, un metabolito, o un estímulo de entrada) ubicado en el eje de las ordenadas con respecto a otro nodo presente en la ruta (que se muestra en el eje de las abscisas). En rojo se muestra la inhibición y, en amarillo, los dos efectos. sus ortólogos. Sin embargo, debido a su posición en la ruta, el efecto de su inhibición podría verse amortiguado por la actividad de la PLC, por lo que no se presentaría un efecto tan drástico según el modelo propuesto. Por otro lado, al comparar la proteína PTEN con la PLC, se encontró que la primera era la que menor porcentaje de identidad exhibía. Al bloquear la acción de la PTEN en una simulación del modelo booleano (figura 2), se alteró la regulación de la producción de los segundos mensajeros PI3P y $\mathrm{PI}, 4,5 \mathrm{P} 3$, y se redujo la producción de PI4P y de PI4,5P2, los cuales desempeñan un papel importante en la ruta, con funciones que van desde la activación de factores de transcripción hasta la formación de vesículas.

Mediante la combinación del análisis de similitud con las proteínas humanas y la simulación del modelo matemático, fue posible proponer la proteína PTEN como el mejor blanco para el desarrollo de medicamentos, tanto por su bajo porcentaje de similitud como por sus efectos drásticos en la ruta de señalización.

\section{Modelo lógico dinámico}

La simulación del modelo booleano en una escala temporal, obtenida con el programa Odefy, permitió determinar los niveles de acumulación de los metabolitos y de las proteínas de la ruta como se ve en la figura 4: en el eje de las ordenadas se muestra cada uno de los nodos y en el eje de las abscisas, la escala temporal. Este ejemplo de simulación de la ruta, se hizo con la misma configuración de los nodos del modelo tardío. Se observa que los perfiles de concentración presentaron un incremento secuencial, según la cercanía a los estímulos externos y los tipos de interacciones que los modulan.

La acumulación de las especies comenzó por el fosfatidil-inoditol, que es el sustrato de las cinasas, y estas empezaron a activarse posteriormente. Después de esta activación, se encontraron los perfiles de las fosfatasas y de los siguientes metabolitos relacionados con los efectos de su activación: IP2, PI, luego DG, CDIPT, seguidos del perfil de IP3, y de IPoIP 1Phos, Myo 1D Mono Phos, para terminar con IP3 Phos I, lo que se explica por su carácter de sustrato, lo cual resulta en una acumulación más lenta.

Después, aparecieron los perfiles asociados con $\mathrm{PI} 3,4,5 \mathrm{P} 3$ y $\mathrm{PI} \mathrm{P}$ (segundos mensajeros importantes de la ruta), cuya activación se mantuvo en niveles bajos debido a que activan e inhiben (es decir, exhiben una activación muy regulada, comportándose a la vez como sustrato y producto de algunas proteínas de la ruta). Por esta razón, el incremento en su concentración depende de los parámetros que caracterizan estas interacciones. También, se presentaron dos perfiles inusuales, correspondientes a PI1D-Myo y a PI4,5P2, que son regulados por un gran número de proteínas cuya activación afecta de manera significativa su producción, mostrando un comportamiento bimodal. 


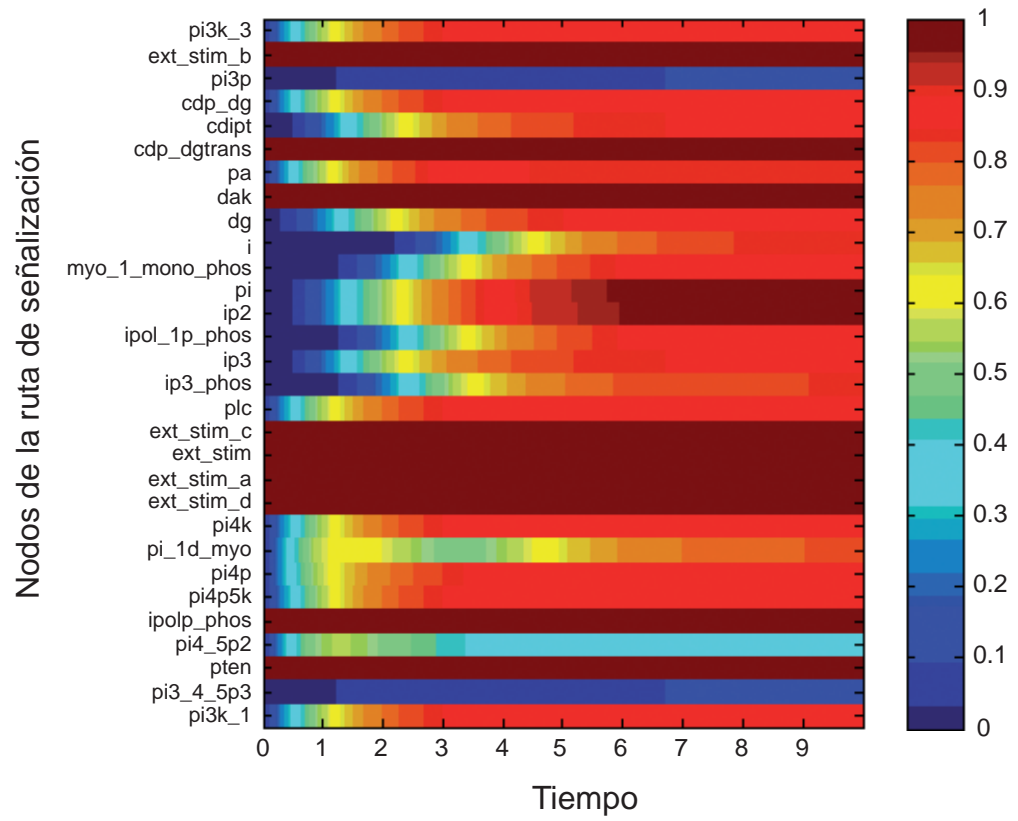

Figura 4. Simulación del modelo lógico dinámico de la ruta del fosfatidil-inositol en Leishmania spp. En el eje de las ordenadas se muestran cada uno de los nodos; en el eje de las abscisas, el tiempo en unidades discretas de las iteraciones del modelo; la escala de colores a la derecha ilustra la actividad normalizada de cada especie.

\section{Discusión}

La ruta de señalización del fosfatidil-inositol es fundamental para múltiples procesos celulares en eucariotas, como la diferenciación, la supervivencia, el transporte intracelular y la activación de factores de transcripción, entre muchos otros $(5,8,9,32-37)$. Este trabajo permitió la reconstrucción de dicha ruta en Leishmania, y constituye una primera aproximación para entender el funcionamiento y las interacciones que subyacen a las proteínas que la conforman y, por lo tanto, a la postulación de nuevos blancos para el desarrollo de medicamentos contra la leishmaniasis. Inicialmente, se identificaron proteínas como las fosfatidil-inositol cinasas, responsables de la producción de segundos mensajeros como el PI3P, el cual se ha asociado con la morfología celular, y la formación de vacuolas y del aparato de Golgi, así como con funciones secretorias y la producción de PI4P y PI3,4,5P3, otros segundos mensajeros que activan la ruta Rho1/Pkc1 a través de las MAP cinasas, regulando la vía PKC y la organización del citoesqueleto $(2,21,29,35)$.

En la ruta también se identificaron fosfatasas como la proteína PTEN, que posee una doble especificidad: desfosforila las proteínas fosforiladas en los residuos tirosina, serina y treonina, y actúa como una lípido-fosfatasa retirando el grupo fosfato de la posición D3 del anillo inositol de varios fosfatidil-inositoles, por lo que es una proteína muy importante para el correcto funcionamiento de la ruta y para su regulación mediante el antagonismo que ejerce en la ruta de señalización PI3K-AKT/ $\mathrm{PKB}$, modulando la progresión del ciclo celular y la supervivencia de la célula (27).

Otras proteínas destacadas en el análisis fueron la INNP y la $\mathrm{PI}(3) 5 \mathrm{PK}$. La primera está involucrada en el metabolismo del inositol para la formación de los fosfatidil-inositoles, pues ayuda a mantener un equilibrio en su concentración a nivel celular. La segunda es la responsable de la formación de $\mathrm{PI}$ 3,5P2, un importante segundo mensajero involucrado en la morfología y la función vacuolar (38).

La ruta de señalización del fosfatidil-inositol produce, además, importantes segundos mensajeros que se han estudiado en otros modelos de organismos. Uno de ellos es el PI4,5P2, sustrato que cataliza PLC para producir DAG e IP3, protagonistas de la activación de liberación del calcio y de la unión a múltiples factores de transcripción (32). También, se ha encontrado que modula la función de algunas proteínas, como las de unión de la actina, con un importante papel en el proceso de exocitosis (32).

Existen, asimismo, otras evidencias que permiten proponer a algunas de las proteínas de la ruta de señalización del fosfatidil-inositol en Leishmania spp. como nuevos blancos terapéuticos y, probablemente, para el desarrollo de vacunas: en primer lugar, la transducción de señales y la invasión del huésped están dirigidas a este grupo de proteínas en Leishmania spp. $(5,36,39-41)$. En segundo 
lugar, ya existen medicamentos (wortmannin, rapamicina) que se utilizan como bloqueadores de la ruta de señalización del fosfatidil-inositol y la vía TOR en otros tripanosomátidos $(9,39)$.

Según el estudio, el sistema de proteínas que constituyen la ruta es sólido. La presencia de múltiples copias de varias de las proteínas evaluadas, da cuenta de la especialización en las funciones, en la que cada proteína cumple unos roles establecidos. Esto indica que, frente a una mutación o alteración en alguna de las copias, la vía no quedará completamente bloqueada, lo cual es crucial dada por su importancia en la supervivencia del parásito. Además, se encontraron ortólogos en el humano para algunas copias, pero no para otras, lo que condujo a que presentaran un porcentaje de similitud diferente cuando se compararon con las proteínas funcionalmente homólogas en el huésped, parámetro que debe ser prioritario a la hora de postular alguna de estas proteínas como blanco para el desarrollo del medicamento.

Entre estas proteínas funcionalmente homólogas, en el grupo de las cinasas se destacan los genes PI4Ky PIK, incluidas proteínas como la ATM, la Atr, la DNA PKC y la TOR, relacionadas con la reparación de daños en el ADN (34) y el crecimiento celular. En el caso de las fosfatasas, se destaca la INNP, cuyos dominios funcionales son muy diferentes a los de las demás fosfatasas, y la IP(3) 5 fos; esto, sumado a sus funciones en la realimentación de la ruta de señalización, las convierte en buenas candidatas a ser blancos terapéuticos, ya que el equilibrio de estas especies es indispensable para el buen funcionamiento de la ruta de señalización (37).

Además de identificar las proteínas pertenecientes a la ruta del fosfatidil-inositol en Leishmania spp., el análisis permitió excluir otras ya descritas en otros organismos, entre ellas, la fosfatidil-inositol 5 cinasa (PI5K), la fosfatidil-inositol 3 cinasa (PI3K) de los tipos $1 \mathrm{~A}$ y $1 \mathrm{~A}, 1 \mathrm{~B}$ y 2 , la fosfatidil-inositol 5 fosfato 4 cinasa (PI5P4K), y la SH2 inositol fosfatasa (SHIP). La ausencia de dichas proteínas descartó la producción de los fosfatidil-inositoles PI5P y PI3,4P2, cuyas funciones aún no se han determinado claramente en los modelos en los que se ha estudiado la ruta, aunque se sabe que pueden estar asociadas con la secreción de neurotransmisores y la regulación de proteínas G (42). Nuestros hallazgos concuerdan con los resultados de otro estudio (5), lo que respalda su exclusión de la red de señalización. Aun así, dicha afirmación requiere una comprobación experimental en este grupo de parásitos.
A partir del modelo matemático, se analizó la ruta mediante simulación computacional y se obtuvieron predicciones sobre su alteración. Con el modelo booleano se demostraron algunas de sus características y se evaluó el efecto de un inhibidor, demostrándose que la ruta es capaz de amortiguar perturbaciones, como se observó cuando la función de la PLC pudo sustituir algunas de las fosfatasas responsables del catabolismo de los fosfatidil-inositoles. Esta proteína esencial para el funcionamiento de la ruta desde el punto de vista del modelo, también posee una función celular imprescindible, como es la formación de PI4,5P2. Además, el modelo permitió llegar a conclusiones sobre la forma en que, a partir de la activación de proteínas como la PLC y la PTEN, la ruta se autorregula y recupera el estado basal. Esta autorregulación se vio afectada al inducir la inhibición de alguna de ellas y permitió observar el efecto de la inhibición de otras proteínas, por ejemplo, el de la IP(3) 5 fos, que aparentemente no es tan drástico según el modelo. De esta manera, estas proteínas se perfilaron como potenciales blancos terapéuticos, a lo cual se suma la evidencia experimental de su papel en otros modelos, como los mamíferos y la levadura.

Con la información obtenida a partir del análisis de similitud con las proteínas humanas, la PTEN se perfiló como un mejor candidato a blanco para el desarrollo de medicamentos. La inhibición de la PTEN evitó que la ruta de señalización regresara a estados basales, lo cual se vio reflejado en una desregulación de los segundos mensajeros $\mathrm{PI}$,5P2, PI3P y $\mathrm{PI} 3,4,5 \mathrm{P} 3$ y, por lo tanto, en múltiples funciones celulares del parásito ya mencionadas que conducirían a un deterioro de la fisiología y la morfología celular, proceso que desencadenaría la muerte del parásito en caso de no existir un mecanismo compensatorio. Sin embargo, es necesario validar esta afirmación experimentalmente.

Cabe aclarar, igualmente, que todas las proteínas mencionadas como posibles blancos servirán de base para la búsqueda de medicamentos cuya eficacia pueda comprobarse experimentalmente, ya que su inhibición o modulación podría provocar un efecto drástico a nivel celular dentro del parásito. No obstante, al ser proteínas que tienen homología con proteínas humanas, incluida la PTEN, es imperativo que se compruebe que los inhibidores diseñados o identificados sean específicos para las diferencias estructurales que tienen con el 
humano. Dicho análisis va más allá del objetivo de este trabajo, por lo que se propone como una perspectiva de investigación futura.

En la segunda parte del modelado, es decir, el modelo dinámico, se evaluaron los niveles de acumulación de los metabolitos en el evento tardío, y se obtuvieron perfiles de concentración temporales de cada uno. Esos perfiles demostraron cómo se produce una acumulación secuencial de los elementos de la ruta de señalización que concuerda con su posición dentro de ella y responde a la forma en que está regulada su producción. En general, se activaron primero las proteínas cinasas y luego las fosfatasas, cuya interrelación da lugar a la producción de los segundos mensajeros que modulan las respuestas celulares frente a la ruta. Aunque este modelo rescata patrones importantes y generales del funcionamiento de la ruta, sería necesario incluir información cinética de la actividad de las proteínas de la ruta, para poder comparar los niveles de producción de cada uno de los segundos mensajeros, por lo que se concluye que este tipo de modelo dinámico tiene mayor valor como perspectiva de investigación.

Este trabajo ofrece una alternativa para el análisis de rutas de señalización sobre las que no existe información suficiente para producir otro tipo de modelos matemáticos, especialmente en estudios de organismos que no constituyen un modelo, ya que permitió predecir los comportamientos y variaciones de la ruta del fosfatidil-inositol en Leishmania spp., con el fin de identificar un posible blanco para el desarrollo de medicamentos y de evaluar su efecto mediante simulación computacional. Asimismo, se abre el camino para ampliar el modelo propuesto para incluir otras proteínas que conecten esta ruta de señalización con otras, y así enriquecer el análisis, y para validar experimentalmente el modelo por medio de estudios de cinética enzimática y de imaginología biológica, entre otros.

\section{Conflicto de intereses}

Los autores declaran que no existe conflicto de intereses en torno al manuscrito.

\section{Financiación}

Este proyecto fue apoyado por la Universidad de Antioquia.

\section{Referencias}

1. Walker RG. Leishmania CRK3: CYC6 cyclin-dependent kinase as a drug target (thesis). Glasgow: University of Glasgow; 2008.
2. El-sayed NM, Myler PJ, Gaëlle L, Mathew B, Crabtree J, Aggarwal G, et al. Comparative genomics of trypanosomatid parasitic protozoa. Science. 2005;309:404-9. http://dx.doi. org/10.1126/science. 1112181

3. Ivens AC, Peacock CS, Worthey EA, Murphy L, Aggarwal G, Berriman M, et al. The genome of the kinetoplastid parasite, Leishmania major. Science. 2005;309:436-42. http://dx.doi.org/10.1126/science. 1112680

4. Ash C, Jasny BR.Trypanosomatid genomes. Science. 2000;309:399-400. http://dx.doi.org/10.1126/science.309. 5733.399

5. Bahia D, Oliveira LM, Lima FM, Oliveira P, Silveira JF Da, Mortara RA, et al.The TryPIKinome of five human pathogenic trypanosomatids: Trypanosoma brucei, Trypanosoma cruzi, Leishmania major, Leishmania braziliensis and Leishmania infantum--new tools for designing specific inhibitors. Biochem Biophys Res. 2009;390:963-70. http://dx.doi.org/10.1016/j. bbrc.2009.10.086

6. Naula C, Parsons M, Mottram JC. Protein kinases as drug targets in trypanosomes and Leishmania. Biochim Biophys Acta. 2005;1754:151-9. http://dx.doi.org/10.1016/j. bbapap.2005.08.018

7. Cohen P. Protein kinases--the major drug targets of the twenty-first century? Nat Rev Drug Discov. 2002;1:309-15. http://dx.doi.org/10.1038/nrd773

8. Stephens L, Williams R, Hawkins P. Phosphoinositide 3-kinases as drug targets in cancer. Curr Opin Pharmacol. 2005;5:357-465. http://dx.doi.org/10.1016/j.coph.2005. 03.002

9. Braga M V, de Souza W. Effects of protein kinase and phosphatidylinositol-3 kinase inhibitors on growth and ultrastructure of Trypanosoma cruzi. FEMS Microbiol Lett. 2006;256:209-16. http://dx.doi.org/10.1111/j.1574-6968.2006. 00125.x

10. Schoijet AC, Miranda K, Girard-Dias W, de Souza W, Flawiá MM, Torres HN, et al. A Trypanosoma cruzi phosphatidylinositol 3-kinase (TcVps34) is involved in osmoregulation and receptor-mediated endocytosis. J Biol Chem. 2008;283:31541-50. http://dx.doi.org/10.1074/jbc. M801367200

11. Lindmo K, Stenmark H. Regulation of membrane traffic by phosphoinositide 3-kinases. J Cell Sci. 2006;119:605-14. http://dx.doi.org/10.1242/jcs.02855

12. Hall BS, Gabernet-Castello C, Voak A, Goulding D, Natesan SK, Field MC. TbVps34, the trypanosome orthologue of Vps34, is required for Golgi complex segregation. J Biol Chem. 2006;281:27600-12. http://dx.doi. org/10.1074/jbc.M602183200

13. Soares MJ. Endocytic portals in Trypanosoma cruzi epimastigote forms. Parasitol Res. 2006;99 4:321-2. http:// dx.doi.org/10.1007/s00436-006-0189-9

14. Kanehisa M, Goto S. KEGG: Kyoto encyclopedia of genes and genomes. Nucleic Acids Res. 2000;28:27-30. http://dx. doi.org/10.1093/nar/28.1.27

15. Myler PJ. Searching the Tritryp genomes for drug targets. Adv Exp Med Biol. 2008;625:133-40. http://dx.doi.org/10. 1007/978-0-387-77570-8 11

16. Altschul SF, Madden TL, Schäffer AA, Zhang J, Zhang Z, Miller W, et al. Gapped BLAST and PSI-BLAST: A new 
generation of protein database search programs. Nucleic Acids Res. 1997;25:3389-402. http://dx.doi.org/10.1093/ nar/25.17.3389

17. Bateman A, Birney E, Cerruti L, Durbin R, Etwiller L, Eddy SR, et al. The Pfam protein families database. Nucleic Acids Res. 2002;30:276-80. http://dx.doi.org/10.1093/ nar/30.1.276

18. Li L, Stoeckert CJ, Roos DS. OrthoMCL: Identification of ortholog groups for eukaryotic genomes. Genome Res. 2003;13:2178-89. http://dx.doi.org/10.1101/gr.1224503

19. Aslett M, Aurrecoechea C, Berriman M, Brestelli J, Brunk BP, Carrington M, et al. TriTrypDB: A functional genomic resource for the Trypanosomatidae. Nucleic Acids Res. 2010;38:D457-62. http://dx.doi.org/10.1093/nar/gkp851

20. Ostlund G, Schmitt T, Forslund K, Köstler T, Messina DN, Roopra S, et al. InParanoid 7: New algorithms and tools for eukaryotic orthology analysis. Nucleic Acids Res. 2010;38:D196-203. http://dx.doi.org/10.1093/nar/gkp931

21. Boratyn GM, Schäffer AA, Agarwala R, Altschul SF, Lipman DJ, Madden TL. Domain enhanced lookup time accelerated BLAST. Biol Direct. 2012;7:12. http://dx.doi. org/10.1186/1745-6150-7-12.

22. Klamt S, Sáez-Rodríguez J, Gilles ED. Structural and functional analysis of cellular networks with CellNetAnalyzer. BMC Syst Biol. 2007;1:2. http://dx.doi.org/10.1186/17520509-1-2

23. Samaga R, Sáez-Rodríguez J, Alexopoulos LG, Sorger PK, Klamt S. The logic of EGFR/ErbB signaling: Theoretical properties and analysis of high-throughput data. PLoS Comput Biol. 2009;5:e1000438. http://dx.doi.org/10.1371/ journal.pcbi.1000438

24. Albert R, Wang RS. Discrete dynamic modeling of cellular signaling networks. Methods Enzymol. 2009;467:281-306. http://dx.doi.org/10.1016/S0076-6879(09)67011-7.

25. Klamt S, Sáez-Rodríguez J, Lindquist JA, Simeoni L, Gilles ED. A methodology for the structural and functional analysis of signaling and regulatory networks. BMC Bioinformatics. 2006;7:56. http://dx.doi.org/10.1186/14712105-7-56

26. Klamt S. Manual CellNetAnalyzer. Magdeburg, Germany: Institute for Dynamics of Complex Technical Systems; 2012.

27. Shibata T, Nishikawa M, Matsuoka S, Ueda M. Modeling the self-organized phosphatidylinositol lipid signaling system in chemotactic cells using quantitative image analysis. J Cell Sci. 2012;125:5138-50. http://dx.doi.org/10.1242/jcs. 108373

28. Oda K, Matsuoka Y, Funahashi A, Kitano H. A comprehensive pathway map of epidermal growth factor receptor signaling. Mol Syst Biol. 2005;1:2005.0010. http://dx.doi. org/10.1038/msb4100014

29. Mueller-Roeber B, Pical C. Inositol phospholipid metabolism in Arabidopsis. Characterized and putative isoforms of inositol phospholipid kinase and phosphoinositide-specific phospholipase C. Plant Physiol. 2002;130:22-46. http://dx. doi.org/10.1104/pp.004770
30. Mirschel S, Steinmetz K, Rempel M, Ginkel M, Gilles ED. PROMOT: Modular modeling for systems biology. Bioinformatics. 2009;25:687-9. http://dx.doi.org/10.1093/ bioinformatics/btp029

31. Krumsiek J, Pölsterl S, Wittmann DM, Theis FJ. Odefy-from discrete to continuous models. BMC Bioinformatics. 2010;11:233. http://dx.doi.org/10.1186/1471-2105-11-233

32. Ishihara H, Shibasaki Y, Kizuki N, Wada T, Yazaki Y, Asano $\mathrm{T}$, et al. Type I phosphatidylinositol-4-phosphate 5-kinases. Cloning of the third isoform and deletion/ substitution analysis of members of this novel lipid kinase family. J Biol Chem. 1998;273:8741-8. http://dx.doi.org/10. 1074/jbc.273.15.8741

33. Leevers SJ, Vanhaesebroeck B, Waterfield MD. Signalling through phosphoinositide 3-kinases: The lipids take centre stage. Curr Opin Cell Biol. 1999;11:219-25. http://dx.doi. org/10.1016/S0955-0674(99)80029-5

34. Bahia D, Oliveira LM, Mortara RA, Ruiz JC. Phosphatidylinositol-and related-kinases: A genome-wide survey of classes and subtypes in the Schistosoma mansoni genome for designing subtype-specific inhibitors. Biochem Biophys Res. 2009;380:525-30. http://dx.doi.org/10.1016/j. bbrc.2009.01.093.

35. Mérida I, Ávila-Flores A, Merino E. Diacylglycerol kinases: At the hub of cell signalling. Biochem J. 2008;409:1-18.

36. Asaki TS, Asaki JS, Akai TS, Akasuga ST, Uzuki AS. Metabolism and functions of phosphoinositides the physiology of phosphoinositides. Society. 2007;30:1599-604.

37. Egea-Jiménez AL, Pérez-Lara A, Corbalán-García S, Gómez-Fernández JC. Phosphatidylinositol 4,5bisphosphate decreases the concentration of $\mathrm{Ca} 2+$, phosphatidylserine and diacylglycerol required for protein kinase $\mathrm{Ca}$ to reach maximum activity. PLoS One. 2013;8 7:e69041. http://dx.doi.org/10.1371/journal.pone.0069041

38. Cooke FT, Dove SK, McEwen RK, Painter G, Holmes AB, Hall MN, et al. The stress-activated phosphatidylinositol 3-phosphate 5-kinase Fab1p is essential for vacuole function in S. cerevisiae. Curr Biol. 1998;8:1219-22. http:// dx.doi.org/10.1016/S0960-9822(07)00513-1

39. Einicker-Lamas M, Nascimento MTC, Masuda CA, Oliveira MM, Caruso-Neves C. Trypanosoma cruzi epimastigotes: Regulation of myo-inositol transport by effectors of protein kinases A and C. Exp Parasitol. 2007;117:171-7. http:// dx.doi.org/10.1016/j.exppara.2007.04.011

40. Mongan TP, Ganapasam S, Hobbs SB, Seyfang A. Substrate specificity of the Leishmania donovani myoinositol transporter: Critical role of inositol C-2, C-3 and C-5 hydroxyl groups. Mol Biochem Parasitol. 2004;135:133-41. http://dx.doi.org/10.1016/j.molbiopara.2004.01.015

41. Gómez MA, Contreras I, Hallé M, Tremblay ML, McMaster RW, Olivier M. Leishmania GP63 alters host signaling through cleavage-activated protein tyrosine phosphatases. Sci Signal. 2009;2:ra58. http://dx.doi.org/10.1126/scisignal. 2000213.

42. Loijens JC, Anderson RA. Type I phosphatidylinositol4-phosphate 5-kinases are distinct members of this novel lipid kinase family. J Biol Chem. 1996;271:32937-43. http:// dx.doi.org/10.1074/jbc.271.51.32937 